La revue La revue pour l'histoire du CNRS

\title{
La nomination de Charles Jacob à la tête du CNRS de Vichy
}

Denis Guthleben

\section{(2) OpenEdition \\ Journals}

Édition électronique

URL : https://journals.openedition.org/histoire-cnrs/1388

DOI : 10.4000/histoire-cnrs. 1388

ISSN : 1955-2408

Éditeur

CNRS Éditions

Édition imprimée

Date de publication : 3 mai 2005

ISBN : 978-2-271-06250-5

ISSN : $1298-9800$

\section{Référence électronique}

Denis Guthleben, «La nomination de Charles Jacob à la tête du CNRS de Vichy ", La revue pour I'histoire du CNRS [En ligne], 12 | 2005, mis en ligne le 03 mai 2007, consulté le 20 mai 2021. URL http://journals.openedition.org/histoire-cnrs/1388 ; DOI : https://doi.org/10.4000/histoire-cnrs. 1388

Ce document a été généré automatiquement le 20 mai 2021.

Comité pour l'histoire du CNRS 


\title{
La nomination de Charles Jacob à la tête du CNRS de Vichy
}

\author{
Denis Guthleben
}

1 Par un arrêté du 8 août 1940, pris à Vichy par le secrétaire d'État à l'Instruction publique et aux Beaux-Arts émile Mireaux, Charles Jacob «est nommé, à titre provisoire, administrateur du Centre National de la Recherche Scientifique» avec «autorité de Directeur du Centre National - Section Recherche pure - et de Directeur du Centre National - Section Recherche appliquée $»^{1}$. La mission qui lui est dévolue consiste à «établir, pour le Ministre de l'Instruction Publique, un rapport sur la situation administrative et financière du Centre, et de proposer toutes mesures concernant l'utilisation du personnel, du matériel et des crédits $»^{2}$. Sa nomination prend effet le 11 août 1940.

2 Le nouvel administrateur du CNRS s'atèle immédiatement à sa tâche : il consulte Aimé Cotton, Émile Borel, Léon Perrier ${ }^{3}$, s'entretient au siège du CNRS avec Georges Jamati, Gabrielle Mineur, rencontre Pierre Auger, Frédéric Joliot, visite des labora

3 toires à Paris, Meudon, Ivry. Il se rend également dès le mois de septembre 1940 à Vichy, où il participe à plusieurs réunions houleuses sur le sort du Centre. Après quat

4 re mois de travail, pendant lesquels il s'est consacré à la fois à la gestion de l'organisme et à la rédaction de son rapport, Charles Jacob remet au nouveau ministre, Georges Ripert, une étude détaillée qui conclut à la nécessité de maintenir et de développer le CNRS : "Méthodiquement dirigé, charpenté et discipliné, ce qui n'est nullement incompatible avec la véritable indépendance scientifique, il peut à sa place et dans le travail, collaborer au relèvement de la patrie $»^{4}$. Les recommandations qu'il y fait sont en partie reprises dans la loi du 10 mars 1941 qui réforme l'organisation de l'établissement public ${ }^{5}$. Charles Jacob est officiellement nommé au poste de directeur le 22 avril 1941', une fonction qu'il quitte à regret à la Libération, en août 1944.

C'est sur les débuts de cette période difficile de l'histoire du CNRS que nous nous proposons de revenir ici. Qui était réellement Charles Jacob? Pourquoi le gouvernement de Vichy a-t-il choisi ce professeur de géologie, académicien depuis 1931, pour remplacer Jean Mercier, un directeur désigné moins de deux mois 
auparavant et dont la fidélité lui était acquise ? Quelle était son opinion sur le Centre et comment a-t-elle évolué ? L'étude d'archives conservées à l'Académie des Sciences et jusqu'alors inexploitées permet de lever un coin du voile. Charles Jacob a gardé à la tête du CNRS les habitudes du géologue, arpenteur infatigable toujours muni d'un carnet et d'un crayon. Il a, au fil des ans, rempli des centaines de pages de notes. Raturées, barrées, fléchées en tout sens, certaines sont illisibles. D'autres sont en revanche d'une grande clarté : elles permettent de mieux comprendre la vie du CNRS sous l'Occupation et de pénétrer la personnalité de son directeur.

«Enfant terrible, vous n'avez pas l'oreille de la grande table !»

6 Charles Jacob n'a jamais fait mystère de son opposition à l'organisation de la recherche inspirée par Jean Perrin dans les années 1930. Nommé en 1938 membre du Conseil supérieur de la recherche scientifique (CSRS), il marque d'emblée son désaccord avec une instance qui «n'a jamais rien exprimé du tout» et n'est qu'une énième manifestation du « virus démocratique » dont la France souffre depuis 1936. Il déplore par-dessus tout la mainmise qu'y exercent son créateur et ses amis, la «bande à Perrin ». Lors de la session du 2 au 5 mars 1938 du CSRS, il participe à une passe d'armes qui ne fait que confirmer ses opinions : « Déjà le matin [...] il y a des incidents de séance et des éclats de voix. Je m'y mêle, peut-être un peu trop; mais comme il n'y a pas lieu d'oublier les réactions du dehors, peut-être est-il bon de faire sentir que tout ne va pas pour le mieux dans le meilleur des mondes». Il quitte la réunion complètement désabusé : "Le passé continue. Que dire à ces gens qui ne veulent rien entendre? ». Jean Perrin a «trop d'amis» dans le milieu scientifique pour que ses détracteurs puissent faire entendre leurs voix et Charles Jacob n'a pas «l'oreille de la grande table", ainsi que le remarque un de ses collègues, Charles Marie, au sortir de la réunion. «Et comment ! », lui répond le géologue ${ }^{7}$.

7 Celui-ci a cependant des idées précises sur ce que devrait être la recherche en France. Il les rédige en détail lors d'un séjour à Mont-Louis, dans les Pyrénées-Orientales, où il s'est réfugié en juillet 1940 après la débâcle. "La croisade de Jean Perrin » n'a abouti qu'à l'instauration d'une «république des camarades» et à la fondation d'un organisme, le CNRS, « indépendant du Directeur de l'Enseignement supérieur », dominé par deux directeurs, géré avec « deux budgets réunissant 110 millions ! », et finalement dirigé « d'une manière plus ou moins occulte par Jean Perrin (sous Léon Blum, c'était un sous-secrétaire d'État et ça voulait le rester !) ». Résultat : « l'accroissement des frais généraux ", " une grosse administration autonome », " pas de coordination » et " des doubles emplois »... bref, « tout est à recharpenter $»^{8}$.

8 Comment ? En rendant à César ce qui, selon Charles Jacob, lui appartient : la recherche doit retourner à l'Enseignement supérieur, qui gérait les laboratoires bien avant la création des «multiples comités » et d'un établissement indépendant comme le CNRS. Il esquisse déjà une organisation d'ensemble : «à côté du directeur de l'Enseignement supérieur, et sous ses ordres, on pourrait avoir un service réduit au point de vue administratif, s'occupant de la distribution de l'argent à la recherche ». Et les laboratoires n'appartenant pas à l'Université ? "Que leurs Ministères les dotent (ils l'ont toujours fait plus ou moins) [...] On demandera toutefois une liaison interministérielle de responsables, qui évitera les doubles emplois et ventilera, renforcera les crédits au mieux du rendement ». Et les disciplines nouvelles? «Créons alors des labos nouveaux; outillons-les, mais rattachons-les administrativement à des organismes existants (Facultés, Collège de France qui est fait pour cela, Muséum, etc.). 
Une direction administrative autonome de la recherche autrement que pour dépenser des crédits est un leurre qui engendre le désordre et prête aux combines ».

Charles Jacob souhaite en fait revenir à un âge d'or mythique de la recherche qu'il évoque avec nostalgie : « à cela, on objecte qu'on va tuer l'indépendance, l'originalité et favoriser les excès des patrons avisés. Ce n'est qu'en partie vrai. Le vieux savant autoritaire, ankylosé ? Les chapelles ? Les «Écoles », soit... Cependant, souhaitons d'en avoir comme au bon vieux temps. Une Inspection générale surveillerait l'indépendance, l'originalité du travail, etc. Toute organisation humaine a des inconvénients. Choisir celle qui a les moindres ». Dans son esprit, le bon vieux temps est celui de «Pasteur et tant d'autres ». De même, pour les recherches appliquées, Charles Jacob estime que les méthodes anciennes ont fait leurs preuves: «Si l'industriel, si l'État, si la Guerre par exemple, veulent s'en donner la peine, ils finiront bien par atteindre l'homme ou le laboratoire qui peut les aider. Combien, en dehors de ma compétence personnelle, en ai-je orienté, en physique, en minéralogie, en chimie, etc. [...] L'organisation des Universités avec les concours matériels qu'elles peuvent accepter, soit de l'État, soit de l'Industrie privée, est assez souple pour permettre cela». En somme, "point n'est besoin d'un organisme central de la recherche ${ }^{9}$.

Le fait de placer un homme aux idées aussi tranchées à la tête du CNRS ne revient-il pas à organiser le sabotage de l'organisme? C'est sans doute ce que souhaitent le gouvernement du maréchal Pétain et son ministre Émile Mireaux qui signe l'arrêté de nomination du 8 août 1940. Mais, contre toute attente, le fossoyeur va se faire avocat et prendre fermement le parti du maintien du Centre.

"Si on veut sauver l'objet dans ce qu'il a d'intéressant... »

11 Comment expliquer ce revirement ? Jusqu'à présent, une thèse a prévalu : Charles Jacob se laisse convaincre, à mesure qu'il avance dans son rapport, rencontre les chercheurs et visite les laboratoires, de la fausseté de ses opinions et de l'utilité du CNRS dans une France défaite, occupée et amoindrie ${ }^{10}$. Les témoignages des acteurs sont venus la conforter : le physicien Jean Wyart, par exemple, explique que «Jacob était un type honnête, son enquête lui avait montré que ses préjugés n'étaient pas fondés, que notamment personne ne s'était enrichi grâce au Centre et il avait rédigé un papier favorable au CNRS $»^{11}$; Gabrielle Mineur, qui dirige le secrétariat du Centre, partage ce point de vue ${ }^{12}$. La réalité serait cependant plus crue.

Le 9 août 1940 à 17 h 30, Jacques Chevalier, secrétaire général du ministère de l'Instruction publique, téléphone à Charles Jacob, qui se trouve dans son laboratoire de géologie à la Sorbonne. Il lui transmet une invitation de Louis Lavelle, directeur du cabinet d'Émile Mireaux, pour le lendemain. Charles Jacob se rend donc au ministère le samedi 10 août à $11 \mathrm{~h}$, où il prend connaissance de l'arrêté de nomination signé à Vichy deux jours plus tôt. Après quelques heures de réflexion, il donne son accord à Louis Lavelle. Le lundi 12 août, Gabrielle Mineur, qui lui a téléphoné la veille pour se mettre à sa disposition, lui fait les honneurs du siège du CNRS, au 13 quai d'Orsay. Dès le lendemain, le mardi 13, le nouveau patron du Centre fait volte-face : «Si on veut sauver l'objet dans ce qu'il a d'intéressant, ne pas commencer à le coller sur la place publique $»^{13}$. Enfin, le mercredi 14 août, Aimé Cotton, qui est venu féliciter Charles Jacob à la Sorbonne, lui remet un rapport mentionnant qu'» au sujet de l'organisation générale de la science il me semble bon qu'un Centre unique s'occupe à la fois des recherches de science pure et de science appliquée». Charles Jacob souligne deux fois 
«Centre unique» et annote dans la marge: «Parfait $»^{14}$. Ses critiques portant sur l'existence d'un « organisme central » sont bien vite oubliées.

Les notes personnelles de Charles Jacob permettent d'apporter deux explications à cette palinodie. La première est implicite : sa nomination est assurément une belle revanche sur la «bande », sur ce "clan des normaliens $»^{15}$ auquel il s'est constamment opposé. La seconde est clairement indiquée dans ses archives: le pouvoir, quel qu'il soit, exerce sur lui une véritable fascination. "Ordre ", "pouvoir fort», "dictature " même, sont les mots qui reviennent le plus fréquemment sous sa plume. Il observe souvent avec une certaine admiration, de la fenêtre de son appartement donnant sur la place du Panthéon, les défilés militaires de la Wehrmacht. Ainsi, le dimanche 10 septembre 1940 : «Je me lève à 8 heures. Pendant que je déjeune passe une compagnie d'Allemands qui défile en chantant devant le lycée Henri IV [...] Tout cela est discipliné, mais lourd. On sent toute une nation commandée, hiérarchisée. L'ordre règne évidemment. Une force - et quelle force - est ainsi obtenue. Force de conquête, force pouvant assurer l'hégémonie d'une race ${ }^{16}$.

14 Les préparatifs de son premier voyage à Vichy font d'ailleurs naître en lui dès la fin du mois de septembre 1940 d'inquiétants rêves de grandeur : «Je ne devrais accepter que le Ministère, avec présence au Conseil des Ministres [...] Mon cabinet, c'est secondaire. Il peut être provisoirement très restreint. Trois ou quatre bons secrétaires et ça suffirait». De l'ambition, tout simplement? Voire. Le directeur provisoire du CNRS souhaite en effet "reprendre en main ce qui reste du pays", conserver le maréchal Pétain parce qu'» on ne peut pas s'en passer » mais en revanche "supprimer Laval» pour garder les mains libres. Il ne lui resterait plus alors qu'à « remettre de l'ordre : la police, l'armée, le ravitaillement, là sont les questions d'une urgence angoissante ${ }^{17}$. Autant de projets qu'il cultive dans le secret de sa conscience jusqu'à la Libération et qui ne trouvent pas même un début d'exécution.

15 Pétainiste, Charles Jacob l'est assurément. Ses notes sur le Maréchal, «ses vues pertinentes, et son langage si objectif, si plein de sens, et si français $»^{18}$, ainsi que sur la situation de la France, gagnée par l'» esprit métèque », victime de la "conception internationaliste $»^{19}$ et minée par «la juiverie, la maçonnerie $»^{20}$, sont sans ambages. Toutefois, son premier séjour à Vichy le déçoit beaucoup, d'une part car le ministère tant convoité n'est pas à l'ordre du jour, d'autre part parce qu'il s'aperçoit que l'entourage du ministre de l'Instruction publique a conservé ses préventions contre le CNRS. Le 28 septembre, il rencontre un « Conseil des Experts » réuni par Georges Ripert et ne peut que constater: "les points de vue de ces messieurs et les miens sont inconciliables $»^{21}$. Qu'importe! Charles Jacob décide de passer outre et de rédiger directement pour le ministre une note prônant la « fusion de la science pure et de la science appliquée », rappelant que le rattachement de la recherche à l'Enseignement supérieur « n'est nullement la solution adoptée à l'étranger ", qu'» il y a tout de même, à la Recherche, des fonctions bien définies, très différentes du fonctionnement en quelque sorte canonique de l'Enseignement supérieur » et concluant finalement au sujet du CNRS «que ce serait une lourde faute de le supprimer et de ne pas lui conserver une certaine autonomie $»^{22}$.

De ce séjour à Vichy, Charles Jacob revient avec un regret et un espoir : regret de ne pas avoir pu rencontrer le maréchal Pétain pour lui faire part de ses vues, mais espoir, toujours, de gravir les marches du pouvoir. Dans cette optique, il compte bien se servir du seul levier qui soit à sa disposition, c'est-à-dire la direction du CNRS : «Si les dieux 
faisaient bien les choses, j'aurais le temps de charpenter la Recherche, de m'y faire la main, de la lancer... le Ministère ne viendrait qu'après $»^{23}$.

«Combien je voudrais m'essayer dans de vraies réalisations... »

17 Il reste deux mois à Charles Jacob pour rédiger son rapport. En octobre et en novembre 1940, son activité est débordante et lui laisse peu de temps libre pour rédiger ses notes personnelles. Jusqu'alors quotidiennes et détaillées, elles deviennent plus espacées et rendent compte de manière laconique de l'emploi du temps chargé de leur auteur. Les sollicitations arrivent de toutes parts : des universitaires qui ont besoin du Centre pour réorganiser leurs laboratoires, des industriels demandant des produits de substitution aux matières premières qui commencent à faire défaut (le cuivre, les combustibles pour moteur), de la Fondation Rockefeller qui souhaite obtenir des précisions sur le CNRS afin de poursuivre son soutien à l'organisme, etc.

Charles Jacob est également soumis aux pressions de Vichy: «Les gens de Vichy voudraient que je fasse vite. Jacob veut faire un monument»; Hélas, ces gens ne comprennent rien. Ce n'est pas un monument que j'envisage, mais récolter un fruit, lorsque j'aurai compris et organisé quelque chose de viable [...] Je me demande si je ne ferai pas mieux de tout envoyer balader [sic]; Je n'en ai pas envie du reste, car la besogne à entreprendre est certaine. Elle dépasse, et comment, un rapport à Vichy ; Combien je voudrais m'essayer dans de vraies réalisations et voir si elles sont possibles dans un pays qui, sans cela, sera fichu. $»^{24}$. Charles Jacob, maître d'œuvre d'une dictature éclairée à la française ? Cela ne se réalise pas. Son rapport, remis en décembre 1940, produit toutefois une bonne impression sur les autorités, qui décident de suivre ses recommandations en maintenant le CNRS et en le réformant quelques mois plus tard selon la plupart de ses suggestions.

Ce rapport n'est pas uniquement consacré au Centre. Charles Jacob y livre sa propre conception de l'ensemble de la recherche scientifique en France : les rapports entre la "science pure" et la "science appliquée", entre la recherche et l'enseignement supérieur, entre les scientifiques et les militaires depuis la Première Guerre mondiale. Il accompagne toutefois ses considérations de propositions de réformes drastiques $\mathrm{du}$ Centre. Un CNRS "méthodiquement dirigé, charpenté et discipliné »" c'est tout d'abord un organisme concentré : «Pour l'avenir, tout ce rapport ne veut plus qu'une seule Direction $»^{26}$. Et cette direction, seule, présiderait aux destinées de l'institution, sans conseils ni comités: "Pour les conseils et comités, je suis de ceux qui estiment que, dans notre pays, et quel que soit le régime politique de demain, il faut en finir avec les Assemblées nombreuses, nommées ou élues, qui [...] interfèrent les unes avec les autres ». Selon l'auteur du rapport, seul le conseil d'administration doit être conservé, pour la simple et bonne raison qu'il est $"$ statutairement obligatoire $»^{27}$. En somme, l'organisation de la recherche voulue par Charles Jacob est à l'image des opinions politiques qu'il expose dans ses notes. Le «virus démocratique» doit être combattu partout.

20 À plusieurs reprises, Charles Jacob revient également sur le statut du personnel. À ce sujet, de la même manière, aucun doute ne subsiste dans son esprit: «Il ne faut pas créer un nouveau corps de fonctionnaires comportant des chercheurs à vie $»^{28}$ et pas davantage "considérer le chercheur comme un individu d'une caste privilégiée " ${ }^{29}$. "On devrait habituer réellement les chercheurs à l'idée que leurs allocations sont temporaires, insiste-t-il, en rapport avec des travaux de recherche, et ne seront maintenues que suivant le rendement de leurs travaux $»^{30}$. Une nécessité, cependant : 
tout le personnel doit être placé sous « l'autorité exclusive » du directeur, qui assumera sa tâche « avec de la méthode et de l'ordre »"

21 Surtout, le CNRS doit rester autonome, en particulier vis-à-vis de l'Enseignement supérieur. Cela se justifie, selon Charles Jacob, «pour deux ordres de raison: d'une part, la fluctuation fatale dans l'attribution des crédits de la Recherche, qui vont souvent bien ailleurs qu'à l'Enseignement supérieur, d'autre part la nécessité d'une collaboration réelle à travers tous les moyens scientifiques du pays $»^{32}$. Autonomie, coordination, gestion de l'ensemble des crédits, que de chemin parcouru par l'ancien contempteur d'un "organisme très lourd d'inspiration démocratique, engagé comme d'autres sur la pente de la démagogie $»^{33}$ !

«Vous y remettrez de l'ordre»

Le rapport convainc les autorités. L'administrateur temporaire y gagne ses galons de directeur, officiellement confirmés en avril 1941, ainsi que l'occasion tant attendue de rencontrer le maréchal. L'entrevue a lieu le 7 janvier 1941, « 5 minutes debout en présence de Chevalier ». Ce ne sont, encore une fois, que des désillusions : "J'entends beaucoup parler de recherche - Oui, M. le Maréchal - Qu'est-ce que la recherche ? Pour le moment, jusqu'ici, la pagaille. Mais avec de l'ordre, ça peut rendre de grands services au pays - Vous y remettrez de l'ordre $\aleph^{34}$. Charles Jacob, déçu, est invité à prendre congé après ce court échange. Il ne perd pas pour autant ses espoirs, qui renaissent à chaque nouvelle convocation de Vichy. Ainsi songe-t-il à nouveau, dès le mois de juin 1941, à son ministère : «Je pêche peut-être par orgueil, par illusion... il me semble cependant que, sans forcer quoi que ce soit, l'heure arrive... Si c'était moi, s'attendre, en tout cas, à des ennuis, à beaucoup de travail... mais l'échéance ne m'effraie pas $»^{35}$.

Dans l'attente d'un avenir qu'il rêve glorieux, Charles Jacob doit toutefois se consacrer à la bonne marche de la maison CNRS. Il est confronté à trois difficultés principales. La première concerne le fonctionnement de l'organisme et le rôle qu'il entend jouer en son sein. Les ralliés de la première heure ont en effet vite déchanté et le directeur se montre de plus en plus excédé par leur comportement : Frédéric Joliot, par exemple, déplore les « lenteurs [de l'administration centrale]... et il pourrait le dire en zone libre. Du chantage, quoi... De la part de ce garçon, qui ne se croit pas de la crotte de bique et exagère dans le sens national et individualiste $»^{36}$. Au siège, la situation n'est guère meilleure: "Mme Mineur, revenue de je ne sais quel voyage en Normandie, fait nettement la gueule et ça ne lui va pas... Le front populaire faisait mieux. Maintenant, ça traîne, etc. [...] M. de Grandsaignes, autre larve, a la prétention larvée d'arrêter [...] Qu'est-ce que c'est que ce pathos, plus exactement tout ce monde, toute cette administration! En définitive, bien des gens (femmes et hommes, femmes surtout, de Grandsaignes y compris) sont à expédier ailleurs. Assez de psychologie! De la véritable administration... et de l'action ! ${ }^{37}$.

La seconde difficulté concerne l'activité scientifique du CNRS. Charles Jacob a en effet la lourde tâche de gérer le passage d'une recherche de guerre à une recherche de subsistance. Bien que ce sujet soit peu développé dans ses notes manuscrites, on imagine qu'il l'a beaucoup occupé jusqu'à la Libération: les rapports détaillés des laboratoires, les demandes adressés par les ministères et les industriels et les comptesrendus de visite sur le terrain qui sont conservés dans ses archives témoignent abondamment en ce sens ${ }^{38}$. 
La dernière difficulté que rencontre Charles Jacob apparait quelques mois plus tard. Elle est liée à la remise en cause de la mission de coordination des recherches par le Centre. Prévue par le décret d'octobre 1939, elle a été confirmée par la loi du 10 mars 1941, qui stipule que l'organisme a pour objet de «coordonner et d'encourager les recherches de sciences pure ou appliquée entreprises par les particuliers et par les services publics, et d'établir une liaison entre les personnes et les organismes qui s'y consacrent $\aleph^{39}$. Or dès le mois d'avril 1942, Charles Jacob apprend avec stupeur que cette mission est remise en cause par une initiative provenant du ministère des Colonies, dirigé par l'amiral Platon. Lors d'un nouveau voyage à Vichy, le directeur du CNRS note ainsi : «Vendredi 3 avril 1942. À 9h30', l'Am. Pl. me reçoit en présence du commandant Chaix. Le Ministre est courtois, mais ferme : "J'aurai ma Direction des recherches, qui, ensuite, s'entendra avec vous [...]». Moi-même, je ne cesse d'y réfléchir. Au fond, je ne suis pas content de l'évolution de cette affaire des Colonies ${ }^{40}$. Les inquiétudes du directeur du Centre sont fondées: malgré tous ses efforts, un Service de recherches scientifiques coloniales est créé par décret le 11 juin 1942 et placé sous la direction du professeur Jeannel, du Muséum d'histoire naturelle. Il s'agit d'une première étape du processus qui aboutit, quelques mois plus tard, à la fondation de l'office de la recherche scientifique coloniale (ORSC, futur Orstom).

Les notes prises par Charles Jacob pendant cette période constituent une source majeure pour les historiens. Elles sont d'autant plus précieuses qu'elles n'ont pas été expurgées et ont été versées telles quelles aux archives de l'Académie des sciences. Toutefois, encore nombreuses jusqu'à la fin de l'année 1941, elles deviennent par la suite plus rares, moins précises. Le directeur du CNRS délaisse en effet de plus en plus ses cahiers. La lourde tâche qui lui incombe, outre les déceptions qu'elle lui procure de plus en plus ${ }^{41}$, ne lui laisse vraisemblablement plus l'occasion de s'y consacrer comme auparavant. En outre, les notes laissent plusieurs sujets en suspens. Elles ne permettent par exemple pas de s'exprimer sur les rapports entretenus entre la direction du CNRS et les Allemands pendant cette période. Il semble, à leur lecture, que ces relations aient été très limitées, Charles Jacob ne mentionnant que trois rencontres : celle avec « un type de la Gestapo militaire » en avril $1941^{42}$, une autre le 9 juillet 1942 avec un représentant du service colonial allemand ${ }^{43}$; enfin, en juin 1943, Charles Jacob est convoqué à la préfecture de police par Der Befehlshaber des Sicherheitspolizei und des $\mathrm{SD}^{44}$ après avoir été anonymement dénoncé comme Juif à cause de son nom de famille. Les remarques s'arrêtent là. Constituent-elles un reflet fidèle de la réalité ? Représentent-elles, au contraire, une vision biaisée, celle d'un directeur qui n'a pas toujours vu ou voulu voir les liens effectivement noués sur le terrain, au niveau des laboratoires? Assurément, cette période de l'histoire du CNRS n'a pas encore livré tous ses secrets.

L'auteur remercie Florence Greffe, Claudine Feuret, Marie-Josèphe Mine et Pierre Leroi, des archives de l'Académie des Sciences, pour l'amabilité de leur accueil et l'efficacité de leur aide. 


\section{NOTES}

1.Arrêté du Ministre-Secrétaire d'État à l'Instruction publique et aux Beaux-Arts, Vichy, 8 août 1940, Archives de l'Académie des sciences (ci-après AAS), fonds Charles Jacob, CNRS II - dossier D 25.

2.Idem.

3.Léon Perrier (1873-1948) préside le conseil d'administration du CNRS. Sénateur radical, il s'abstient de voter les pleins pouvoirs au maréchal Pétain lors du vote du 10 juillet 1940, ce qui lui vaut d'être relevé de ses fonctions et remplacé par M. RouchonMazerat.

4.Rapport à M. le Ministre de l'Instruction Publique sur le Centre National de la Recherche Scientifique par M. Charles Jacob, de l'Académie des Sciences, Professeur à la Faculté des Sciences de Paris, décembre 1940, p. 85, AAS, fonds Charles Jacob, CNRS IV - dossier D 27. 5.Loi n 1069 du 10 mars 1941 relative au Centre national de la recherche scientifique parue dans le Journal officiel de l'état français du 28 mars 1941, p. 1330.

6.Décret $n^{\circ} 1778$ du 22 avril 1941 portant nomination du directeur du Centre national de la recherche scientifique publié dans le Journal officiel de l'état français du 16 juin 1941, p. 2509.

7.AAS, fonds Charles Jacob, CNRS I - dossier D 24 pour les citations de ce paragraphe provenant des notes manuscrites de Charles Jacob prises lors de la session du CSRS.

8. « Mes réflexions pendant l'exode après la débâcle », AAS, fonds Charles Jacob, CNRS II - dossier D 25, pour les citations de ce paragraphe (notes manuscrites rédigées dans le courant du mois de juillet 1940).

9.Idem.

10.Voir, par exemple, Jean-François Picard, La République des savants. La recherche française et le CNRS, Paris, Flammarion, 1990, p. 73-74.

11.Témoignage de Jean Wyart publié dans les Cahiers pour l'histoire du CNRS, 2, 1989, p.

22.

12.Témoignage de Gabrielle Mineur, ibid., p. 39.

13. « Le 9 août 1940 - 17h30'. Le coup de téléphone de Lavelle et sa suite - 20 août », AAS, fonds Charles Jacob, CNRS II - dossier D 25 (notes manuscrites).

14. « Le Professeur A. Cotton, directeur [du laboratoire des basses températures et de l'électro-aimant de Bellevue] à Monsieur le Directeur du Centre de la Recherche Scientifique ", AAS, fonds Charles Jacob, CNRS II - dossier D 25. Le rapport est daté du 4 août 1940, soit quatre jours avant la nomination de Charles Jacob. Il était donc sans doute destiné, à l'origine, à son prédécesseur Jean Mercier.

15. « Le 9 août 1940 - 17h30'. Le coup de téléphone de Lavelle et sa suite - 20 août », op. cit.

16." Septembre 1940 et 1er voyage à Vichy ", AAS, fonds Charles Jacob, CNRS II dossier D 25.

17.Idem. Ces notes ont été rédigées entre le 25 et le 28 septembre 1940, à la veille du départ de Charles Jacob pour Vichy.

18.Notes rédigées le « 4 juin 1941, 23 heures », AAS, fonds Charles Jacob, CNRS III dossier D 26.

19.« Mes réflexions pendant l'exode après la débâcle », op. cit.

20.Notes rédigées le « 4 juin 1941, 23 heures », op. cit. 
21. "Septembre 1940 et $1^{\text {er }}$ voyage à Vichy », op. cit.

22. Notes pour Monsieur le Ministre sur la Recherche Scientifique », AAS, fonds

Charles Jacob, CNRS II - dossier D 25.

23. "Septembre 1940 et 1er voyage à Vichy », op. cit.

24. "Octobre 1940 », AAS, fonds Charles Jacob, CNRS II - dossier D 25.

25.Rapport à $\mathrm{M}$. le Ministre de l'Instruction Publique sur le Centre National de la

Recherche Scientifique par M. Charles Jacob, op. cit., p. 85.

26.Ibid., p. 78.

27.Ibid., p. 80.

28.Ibid., p. 6 .

29.Ibid., p. 7.

30.Ibid., p. 55.

31.Ibid., p. 11.

32.Ibid., p. 16.

33. "Mes réflexions pendant l'exode après la débâcle ", op. cit., note manuscrite rédigée dans le courant du mois de juillet 1940 .

34. « Janvier à avril $1941 »$, AAS, fonds Charles Jacob, CNRS III - dossier D 26.

35. « 23 juin - 3 juillet 1941, $3^{\mathrm{e}}$ voyage en zone libre », AAS, fonds Charles Jacob, CNRS III - dossier D 26.

36. "Octobre $1940 »$, op. cit.

37. « Après-midi du 21 mai ", AAS, CNRS III - dossier D 26. M. De Grandsaignes avait en charge la gestion financière du CNRS.

38. L'analyse de ces documents permettra d'ailleurs au Comité pour l'histoire du CNRS de dresser un état des lieux des recherches effectuées au sein de l'organisme pendant la période de l'Occupation.

39. Loi $\mathrm{n}^{\circ} 1069$ du 10 mars 1941, op. cit.

40.« 1942 : janvier à mai », AAS, fonds Charles Jacob, CNRS III - dossier D 26.

41. Mon travail ? Un peu décevant. Ça n'aboutit guère. Tout au plus, je maintiens.

J'assure du service courant. Je distribue des pourboires, au mieux », écrit-il par exemple le 4 mars 1943, « 1943 », AAS, fonds Charles Jacob, CNRS III - dossier D 26.

42. "Janvier à avril 1941 », op. cit. Le dimanche 6 avril, Charles Jacob note ainsi : «Un type de la Gestapo militaire ne convenait-il pas dans mon bureau, il y a quelques jours, qu'on ne pourra pas gouverner et travailler à Paris, à Alger, etc... comme à Berlin ? » 43. " 1942 : juin à septembre », op. cit. Charles Jacob précise que Conrad de Sahmen, du service colonial du Reich, veut établir des « relations avec le monde scientifique français ».

44. " 1943 », op. cit. Il s'agit du commandant territorial SS de la police de sécurité et du service de sécurité (SD : Sicherheitsdienstes) de Paris, Helmut Knochen.

INDEX

Mots-clés : CNRS, Charles Jacob, CNRS de Vichy, Vichy 
AUTEUR

DENIS GUTHLEBEN

Denis Guthleben est assistant scientifique auprès du Comité pour l'histoire du CNRS. Il termine une thèse sur l'image des États-Unis à la télévision française à l'université de Paris I. 